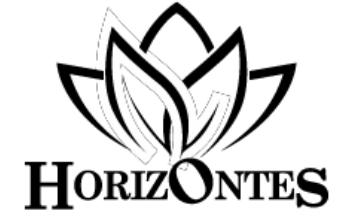

www.revistahorizontes.org
Horizontes. Revista de Investigación en Ciencias de la Educación Https://doi.org/10.33996/revistahorizontes.v2i7.55 julio - septiembre, 2018 Volumen 2 / No. 7 pp. 181 - 190

\title{
Diseño de un curso virtual bajo la plataforma MOODLE. Una visión del autismo desde la Promoción de la Salud
}

\author{
Design of a virtual course under the MOODLE platform. A view of autism \\ from Health Promotion
}

\author{
Alena García \\ alenarosaster@gmail.com \\ Universidad Pedagógica Experimental Libertador. Instituto Pedagógico de Caracas, Venezuela
}

\section{RESUMEN}

El presente artículo describe el diseño de un prototipo partiendo del modelo de desarrollo instruccional propuesto por Müller y Szczureck (1989). Se centra en la necesidad de incorporar un tema novedoso en la formación del futuro docente de Educación Especial de la UPEL - IPC. Es un proyecto factible apoyado en una investigación documental. Se asumió el modelo de Dorrego y García (1993) para la producción de medios instruccionales. El objetivo general es: Promover un espacio virtual de aprendizaje para dar a conocer el autismo como parte de la condición del ser humano y su abordaje desde la promoción de la salud. Los objetivos específicos son: a) Analizar el desarrollo humano y los procesos que intervienen en su evolución, b) Conocer la discapacidad como parte de las alteraciones del desarrollo humano y c) Estudiar el autismo como parte de un espectro y su abordaje interdisciplinario desde la promoción de la salud. Como conclusión se obtiene que el autismo es un tema que puede ser dado a conocer a través de cursos on - line a mayor cantidad de personas, en un formato mucho más flexible e innovador.

Palabras clave: prototipo; desarrollo instruccional; autismo; promoción de la salud

\begin{abstract}
This article describes the design about a prototype based on the model of instructional development proposed by Müller and Szczureck (1989) it focuses on the need to incorporate a new topic in the training of future teachers in Special Education at the UPEL - IPC. It is a feasible project supported by documentary research. Was assumed with the similar form used by Dorrego and Garcia, (1993) to the production of instructional media, the general objective is: To promote space with a virtual learning, to show autism as part of the human condition from the health promotion. The specific objectives are: a) To analyze human development and the processes involved in their development, b) Knowing disability as part of changes in the human development C) Study autism as part of a spectrum and its interdisciplinary approach from promotion of the health. In conclusion the Autism can be known through online courses in a flexible and innovative format.
\end{abstract}

Key words: prototype; instructional development; autism; Health promotion 


\section{INTRODUCCIÓN}

El avance vertiginoso de las Tecnologías de Información y Comunicación (TIC) con el advenimiento del siglo XXI ha llevado a redimensionar el rol del docente, de uno pasivo a otro completamente activo, en el cual se adentra en la nueva era del conocimiento y trasciende de la presencialidad a los espacios virtuales de aprendizaje. .Este profesional pone en práctica un conjunto de estrategias para mediar el proceso de la instrucción en espacios que motiven la participación del grupo, respetando su diversidad o diferencias individuales. Adicionalmente, el profesional de la docencia se encuentra en permanente actualización con los avances de la ciencia y las formas de comunicarse que existen hoy en día, tal como el uso de las redes sociales, en conjunto con las herramientas web, que brindan valiosos insumos para gestionar el conocimiento.

De igual manera, en Venezuela la dinámica de hoy en día dentro en el contexto universitario requiere que tanto los estudiantes como los profesores de todas las disciplinas se comuniquen y generen nuevas formas de interacción en la que se potencien los saberes. Estas pueden desarrollarse en entornos de aprendizaje presenciales, semi presenciales o virtuales, haciendo uso de plataformas tecnológicas mucho más amigables y flexibles en cuanto a diseño y formas de interacción.

Por ende, surgió el interés por parte de las autoras de diseñar un prototipo con objetivos instruccionales, vinculado con la temática del autismo, desde la promoción de la salud, debido a la trayectoria de una de ellas en este ámbito y su interés por darle continuidad, con el asesoramiento de la Doctora en Educación de la Universidad Pedagógica Experimental Libertador (UPEL) y Magíster en Tecnología y Desarrollo de la Instrucción.

Es importante señalar que la autora del prototipo del curso es profesora de Educación
Especial en Retardo Mental, Magíster en Tecnología y Desarrollo de la Instrucción y desde el año 2002 se ha preocupado por investigar en el área del autismo. Ha tenido experiencia en dos instituciones educativas que brindan atención a educandos con esta condición (Centro de Atención Integral para Personas con Autismo, CAIPA y Centro Integral para la Comunicación Cognitiva y Comunicativa, CIPECC). A partir de allí, se interesó por formarse en el área, motivándose a realizar un diplomado en autismo en la Universidad Monteávila. Con el transcurrir del tiempo, en su observación participante dentro de los centros de práctica como docente de la UPEL en el Instituto Pedagógico de Caracas (IPC) se ha percatado de la alta incidencia de este tipo de trastorno en las unidades operativas pertenecientes a la modalidad de Educación Especial y además existen niveles de compromiso desde los más graves hasta los más leves, estos últimos frecuentemente se encuentran en escuelas regulares. Adicionalmente, existe un alto número de estudiantes con autismo que presenta compromiso cognitivo y de tal manera se asocia a la Discapacidad Intelectual.

Actualmente el autismo se ha convertido en un trastorno que se incluye en el Espectro Autista. Los Trastornos del Espectro Autista (TEA) son aquellos que impactan al desarrollo normal del cerebro en áreas relacionadas con la interacción social y las habilidades comunicativas.Se estima que la proporción de personas dentro del TEA es de seis de cada mil y afecta cuatro veces más a los hombres que a las mujeres (East y Evans, 2013). En resumen, el autismo es un trastorno que interfiere en el curso del desarrollo evolutivo del ser humano que conlleva ciertas características particulares en las formas de relación social y que puede asociarse o no a otros cuadros. Forma parte de la condición humana y requiere un abordaje multidisciplinario para potenciar las capacidades. 
Actualmente, tanto en el ámbito internacional como en el nacional existen varias asociaciones y fundaciones lideradas por padres $\mathrm{y}$ profesionales encargados de brindar una atención integral a las personas con autismo a lo largo de su vida, promoviendo su inclusión social. Como parte de su experiencia en el área de evaluación y orientación, los miembros de la fundación autismo en voz alta han generado importantes contribuciones en el estudio de los TEA y a propósito es conveniente citar el siguiente aporte:

El autismo es un trastorno relacionado con limitaciones sociales y de comunicación que se presenta con distintos grados de severidad y es por ello que se habla de los TEA. Es muy diverso; cada persona con autismo es única en sus destrezas, habilidades, talentos y síntomas. Los TEA pueden ser difíciles de diagnosticar, porque no existen pruebas médicas, como los análisis de sangre, para diagnosticar estos trastornos. $\mathrm{Pa}$ ra hacer un diagnóstico, los especialistas evalúan la conducta de la persona y su desarrollo. Las investigaciones indican que con una intervención temprana, en un entorno educativo apropiado, se pueden lograr avances significativos en el desarrollo de destrezas y habilidades de la persona con autismo

(http://autismoenvozalta.com)

Tal como lo señala el texto anterior, los TEA forman parte de una situación bastante compleja y por tal motivo es necesario que los profesionales reciban algún tipo de información que garantice la mejora en la atención temprana u oportuna para la prosecución de una atención educativa integral de calidad. En cuanto a las causas que potencian el autismo, es importante señalar que son muchas y des- de el punto de vista ambiental pueden existir ciertos desencadenantes que conduzcan a mayor predisposición en la aparición del trastorno, el cual se manifiesta durante los tres primeros años de vida, tales como la intoxicación por metales pesados o exposición por parte de la madre durante la gestación a agentes químicos. Por tales razones, surge la inquietud de investigar y dar a conocer el tema del autismo visto desde la promoción de la salud, con el fin de que todo docente pueda poner en práctica esta información en sí mismo y en su praxis educativa, más aún si encuentra en su entorno de aprendizaje a una persona con este tipo de trastorno.

Son muchos los trabajos de investigación que desde hace cinco años hasta la fecha han sido realizados por estudiantes de las especialidades de Educación Inicial, Dificultades de Aprendizaje y Retardo Mental en su fase de ejecución de proyecto educativo de la UPEL IPC del diseño curricular de 1996 que han sido conocidos por la autora en las jornadas de presentación al finalizar cada semestre de pregrado, al mismo tiempo ha sido tutora de un trabajo enmarcado en el área de autismo y la orientación de la misma universidad y también de la Monteávila. Los trabajos han surgido debido a que los investigadores en su experiencia educativa se han encontrado con educandos que presentan esta condición y despierta su inquietud por indagar en esta área de conocimiento, para promover propuestas que sean viables de ejecutar en mejora de la calidad educativa.

Generalmente, los docentes en su práctica o en su ejercicio profesional declaran cierto desconcierto cuando en su espacio de trabajo se consiguen con una persona que presente esta particularidad y frecuentemente sienten miedo e inseguridad al momento de impartir su conocimiento, por desconocer la respuesta o comportamiento que pueda manifestar el estudiante con autismo. Por ende, es necesa- 
rio que tanto ellos como estudiantes de otras especialidades de la UPEL - IPC conozcan acerca de la temática, con el fin de actualizar su formación de forma integral en un campo desconocido, pero que hoy en día es más común que antes, asumiéndolo como parte de la condición humana. Esta se caracteriza por una gran diversidad con respecto al comportamiento y relación social, vinculándose con otras dimensiones importantes como el ambiente y la salud.

El autismo es un tema que guarda relación directa con el ambiente debido a que se involucra con las relaciones humanas, la protección de los derechos humanos y la calidad de vida o bienestar que se logra en la medida en la que se garanticen las condiciones vinculadas con la salud y su promoción. Al respecto, se adopta el aporte de la Organización Mundial de la Salud (1998):

Constituye un escalón más dentro del proceso de atención integral, que viene definido por: la asistencia (primaria y especializada), la prevención (primaria, secundaria y terciaria), la adaptación social a un problema crónico (rehabilitación, cuidados, integración) y finalmente la promoción de la salud (referida a la implicación de los individuos en el desarrollo y disfrute de su salud).

La promoción de la salud se comprende como el conjunto de acciones intersectoriales dirigidas a la comunidad para enaltecer sus derechos y garantizar la protección de su bienestar, a lo largo de su vida. Requiere la participación de los profesionales de la educación para llevarse a cabo en sus tres niveles, en correspondencia con las necesidades que presentan los miembros de una comunidad y los servicios disponibles que pueden ser utilizados, con el fin de satisfacerlas.
La promoción de la salud es un tema que debe ser incorporado en la educación debido a que se establece como un proceso que requiere de la participación de todos los sectores para promover el bienestar de los seres humanos. Está respaldada por organismos internacionales tales como la UNESCO (Organización de las Naciones Unidas para la Educación, la Ciencia y la Cultura) la ONU (Organización de las Naciones Unidas) la Organización Mundial de la Salud (OMS) y la UNICEF (Fondo Internacional de Emergencia de las Naciones Unidas para la Infancia).

La promoción de la salud, al ser abordada desde la educación según Gavidia (2003) se encamina hacia el siguiente propósito:

La promoción de la salud a través de la educación formal tiene especial importancia, y agrega, que la concepción o verdad del concepto de salud que maneje el docente, influirá de forma directa en la manera como facilita la educación para la salud en sus educandos: con enfoque parcelado, totalizador o complejo (p. 1).

La promoción de la salud entonces es uno de los eslabones fundamentales en la formación y en la praxis docente, a través del cual se pueden abordar los trastornos que interfieren en el desarrollo humano, haciendo particular énfasis en el autismo, como ocurre con el siguiente trabajo. En la medida en que el docente se empodere de mayor información al respecto, se garantizarían mayores acciones concretas conducentes a la mejora de la calidad de vida de los estudiantes y hoy en día se puede brindar este conocimiento de una forma asincrónica como se hace con el uso de las plataformas virtuales en este caso la denominada como MOODLE que complementen la administración del currículo de las Especialidades impartidas en la UPEL. 
De forma complementaria, es importante añadir que el currículo es concebido como espacio público donde se interrelacionen los ejes transversales dentro de los cuales se menciona el eje investigación y el de tecnología dentro del Diseño Curricular 2015 de la UPEL, a partir de los cuales se puede incorporar al autismo en la formación del docente, desde la promoción de la salud. Adicionalmente, es necesario considerar el diseño del prototipo bajo la modalidad de educación a distancia para dar respuesta a las demandas de la sociedad y haciendo uso de las TIC Según las ideas de Lozano y Burgos (2010):

La educación a distancia ocurre cuando el profesor y el estudiante no se encuentran físicamente en el mismo lugar y no necesariamente al mismo tiempo. En la educación a distancia, la comunicación necesaria entre el profesor y el estudiante se puede dar por varios medios tecnológicos, tales como el uso de impresos enviados por correo postal, el teléfono, el fax y, más recientemente, por el uso de tecnologías basadas en Internet; eventualmente se dan encuentros presenciales entre profesores y alumnos. (p. 23).

Adicionalmente, en el contexto nacional se establece desde el año 2006 la Ley Orgánica de Ciencia, Tecnología e Innovación, de allí la importancia de incorporar este tema dentro de la formación docente. En su artículo 5 menciona lo siguiente:

Las actividades de ciencia, tecnología, innovación y sus aplicaciones, así como, la utilización de los resultados, deben estar encaminadas a contribuir con el bienestar de la humanidad, la reducción de la pobreza, el respeto a la dignidad, a los derechos humanos y la preservación del ambiente (p. 6).
Hoy en día la educación a distancia se ha convertido en una modalidad de aprendizaje que conlleva un gran impacto social en la sociedad. Según Moore y Kearsley (1996):
La educación a distancia es un aprendizaje planeado que normal- mente ocurre en un lugar diferente del de la enseñanza y como resul- tado requiere técnicas de diseño de cursos especiales, técnicas instruc- cionales especiales, métodos de comunicación electrónicos o basa- dos en tecnologías especiales, así como arreglos administrativos $\mathrm{y}$ organizacionales especiales (p. 2).

Se pone en evidencia entonces que el diseño e implementación de un curso bajo la plataforma virtual se justifica en el ámbito legal y social de una forma contundente y además, responde a las demandas actuales de la sociedad, como uno de los principios que son desarrollados como parte de la educación universitaria.

De acuerdo a lo señalado anteriormente, es válido mencionar que el presente estudio tuvo como propósito promover un espacio virtual de aprendizaje para dar a conocer el autismo como parte de la condición del ser humano y su abordaje desde la promoción de la salud. Además Analizar el desarrollo humano y los procesos que intervienen en su evolución. Conocer la discapacidad como parte de las alteraciones del desarrollo humano. La promoción de la salud. Desde su abordaje interdisciplinario. Y por último Estudiar el autismo como parte de un espectro y su abordaje interdisciplinario desde la promoción de la salud.

\section{MÉTODO}

Este trabajo se circunscribe en la modalidad de proyecto factible que según la UPEL (2016): “consiste en la investigación, elabora- 
ción y desarrollo de una propuesta de un modelo operativo viable para solucionar problemas, requerimientos o necesidades de organizaciones o grupos sociales; puede referirse a la formulación de políticas, programas, tecnologías, métodos o procesos". (p. 21). Este trabajo se corresponde con la metodología descrita debido a que pretende satisfacer las necesidades de formación del futuro docente especialista de la UPEL - IPC en el área de autismo desde la promoción de la salud, con el diseño de un curso bajo la plataforma MOODLE.

Al mismo tiempo, el trabajo deriva de una investigación documental, definida como: "el estudio de problemas con el propósito de ampliar y profundizar el conocimiento de su naturaleza, con apoyo, principalmente, en trabajos previos, información y datos divulgados por medios impresos, audiovisuales o electrónicos". (ob. cit.) (p. 20). Para dar cumplimiento a esta definición, el contenido del curso proviene de una indagación exhaustiva por parte de la autora en diferentes fuentes bibliográficas y resultados de búsqueda por internet, con el fin de complementar los contenidos a ser desarrollados en cada unidad.

Para cumplir con esta meta, fue considerado el modelo de desarrollo instruccional de Müller y Szczureck (1989). El mismo establece las siguientes etapas: Estudio de necesidades, diseño de la solución, la implantación de la solución y evaluación, tal como lo muestra el siguiente gráfico:

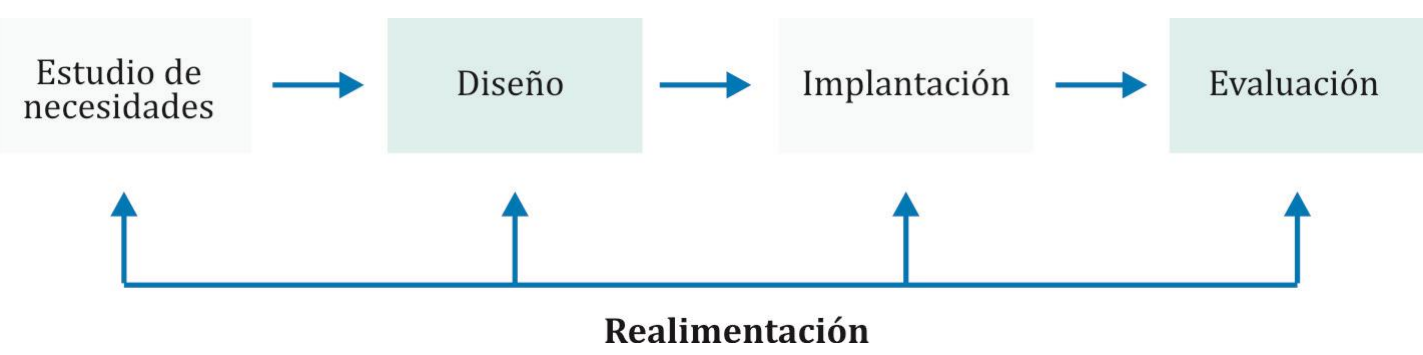

Gráfico 1. Modelo de Desarrollo Instruccional propuesto por Müller y Szczurek, 1989

En la primera etapa, referida al estudio de necesidades se hizo la identificación de la situación, que en este caso es la ausencia o poca formación en el área de la promoción de la salud vinculada con el autismo, al revisar el Diseño Curricular de 1996 de la UPEL de la Especialidad de Educación Especial de las tres Especialidades (Deficiencias Auditivas, Dificultades de Aprendizaje y Retardo Mental). El estudio de necesidades fue realizado a través de la observación participante y la experiencia de la autora dentro del sistema objeto de análisis.

\section{Enunciado de la necesidad}

Partiendo del estudio de necesidades realizado por la autora, derivaron las siguientes necesidades:

1. Carencia de cursos que brinden información acerca del autismo en la Especialidad de Dificultades de Aprendizaje y Deficiencias Auditivas del IPC del Diseño Curricular de 1996 de la UPEL.

2. En el resto de las especialidades no se brinda ningún curso obligatorio u optativo vinculado al tema del autismo.

3. A pesar de la existencia del curso de autismo como optativo de profundización para la especialidad de Educación Especial en Retardo Mental del Diseño Curricular de 
1996 de la UPEL, el mismo se desarrolla con énfasis en la condición, dejando a un lado su abordaje desde la promoción de la salud.

\section{Alternativas de solución}

1. Diseño de un curso dentro de las UNCLE (Unidades Curriculares de Libre Elección) en el marco de la transformación curricular de la UPEL, con el Diseño Curricular 2015, que se pueda impartir a todas las especialidades.

2. Incorporar en el Diseño Curricular 2015 de la UPEL cursos que vinculen al estudiante con la realidad social del país en el componente de formación contextualizada, bajo los principios de la Promoción de la Salud y los niveles de actuación.

3. Incorporar en el servicio comunitario: Prevención primaria de la discapacidad en la comunidad administrado por una de las autoras del presente artículo la temática del autismo y su abordaje desde la promoción de la salud.

4. Diseño e implementación de un curso bajo la plataforma MOODLE en el salón virtual de la UPEL - IPC.

Las alterativas mencionadas son factibles, pero en función del tiempo la más viable y que se puede concretar en menor cantidad de tiempo es la número 3 con el fin de que el curso on - line pueda servir para el desarrollo de las otras vías de solución planteadas.
En cuanto a la etapa de diseño, se consideró viable el diseño de un prototipo en un formato que sea de difusión social al cual pueda acceder gran cantidad de personas sin restricciones en cuanto al tiempo, formación académica o conocimientos previos. Por ende, se diseñó un curso bajo la plataforma MOODLE, definida como:

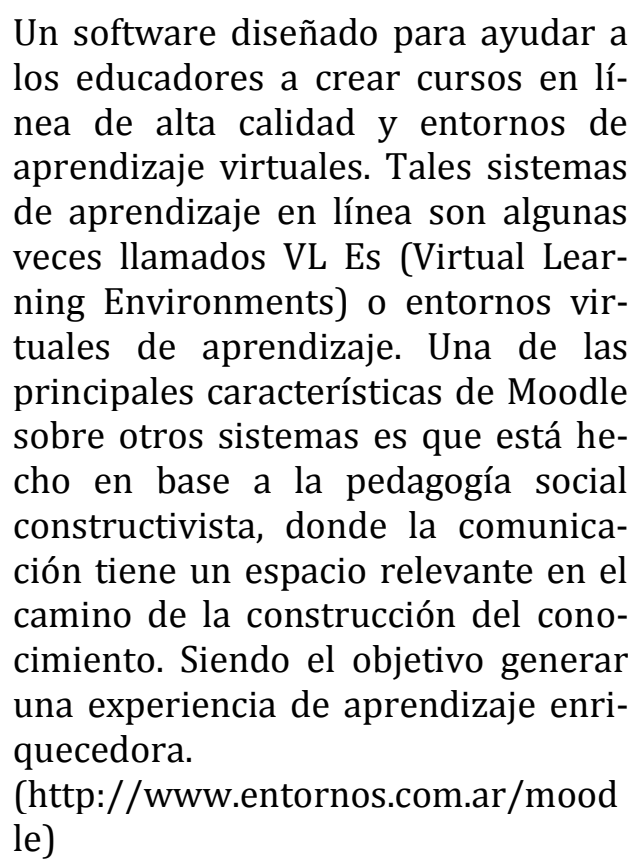

Además, se tomaron en cuenta las fases en la producción de medios instruccionales propuestas por Dorrego y García (1993): planificación, realización y evaluación, tal como se muestran en el siguiente gráfico:

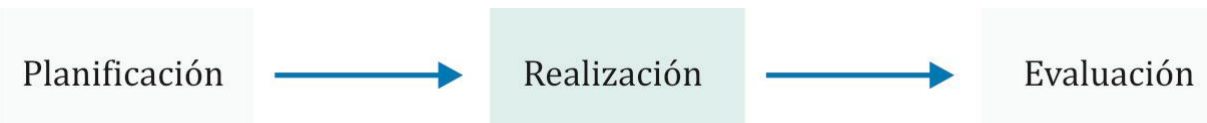

Gráfico 2. Etapas en la producción de medios instruccionales, Dorrego y García (1993)

Según las autoras, la etapa de planificación de una material instruccional comprende el plan didáctico y el plan de producción. Para efectos del prototipo que fue elaborado, se realizó un guion de contenido y un guion técnico - didáctico. El primero contiene el tipo de recurso, área de conocimiento, denominación, propósitos, competencias, unidades a desa- 
rrollar junto a los contenidos, metodología y referencias. Partiendo del estudio de necesidades, la autora consideró factible el diseño de un curso dentro de la plataforma MOODLE del Instituto Pedagógico de Caracas (Salón virtual de la Universidad Pedagógica Experimental Libertador). El mismo fue denominado: EL AUTISMO. UNA VISIÓN DESDE LA PROMOCIÓN DE LA SALUD.

En cuanto al guion técnico - didáctico, se elaboró un esquema que incluye en forma descendente cada una de las secciones que se encontrarán en el curso, incluyendo el guion narrativo y el icónico en cada apartado. A continuación, se describen los objetivos del curso:

En cuanto a la etapa de realización, con el apoyo del guion técnico - didáctico la autora comenzó a cargar el contenido en cada una de las secciones dentro de la plataforma. Al mismo tiempo, hizo uso de programas como creación de issuu, voky y slideshare para almacenar información de una forma más atractiva e innovadora.

Con respecto a la evaluación, el curso fue validado por un juicio de expertos (dos profesores del área de tecnología educativa y otro del área de autismo), quienes tuvieron la posibilidad de entrar como invitados o participantes del curso, con el fin de emitir su juicio en cuanto al diseño, formato y estructura de las unidades y los recursos de apoyo.

De igual forma, el curso fue administrado en el semestre 2016 - II en el cual participó un grupo de 12 estudiantes de Educación Especial en Retardo Mental del Diseño Curricular 1996 de la UPEL. Al finalizar las sesiones se les envío un formato de evaluación para que fuses llenado de forma personal y según su criterio pudiese evaluar el curso en cuanto a los siguientes aspectos: Contenidos, desempeño del facilitador y desempeño del participante.
RESULTADOS

La formación del docente especialista de la UPEL - IPC se encuentra en un proceso de revisión por parte de los profesionales para dar respuesta a las demandas actuales de la sociedad y una de las vías a través de las cuales se puede mejorar la praxis docente es la incorporación de las TIC al sistema instruccional tal como se hizo en el trabajo citado en el presente artículo. Como resultado se obtuvo que el uso de las TIC es un recurso indispensable para promover la adquisición de los aprendizajes significativos en los estudiantes. Durante la evaluación del curso por parte de los participantes se obtuvo como resultado que un $80 \%$ de los participantes indicaron sus respuestas hacia la calificación de excelente y el otro $20 \%$ en el rango de muy bueno, en cuanto a los contenidos, el desempeño del facilitador y el del participante.

Uno de los comentarios que se obtuvo de los estudiantes y se considera pertinente de mencionar es el siguiente: ...En lo personal considero que fue una experiencia diferente a las demás materias ya que fue muy productivo los temas vistos en clase, considero que la información quedo y más con la fluidez que caracteriza a la profe Alena... Estos resultados positivos motivan al docente para continuar con su labor de formar a los estudiantes que son el futuro del país, quienes se convertirán en transmisores de conocimientos y fomentadores de valores en las futuras generaciones.

\section{CONCLUSIÓN}

Con el diseño de este curso, la autora pudo reafirmar la necesidad de incorporar en la formación docente temas tan importantes como el del autismo y la promoción de la salud, que en algunos momentos son ignorados en el resto de las especialidades, con el fin de propiciar mayores espacios de inclusión en el momento en que más individuos conozcan cómo abordar a las personas que presentan esta

condición. 
Un curso bajo la plataforma MOODLE se convierte en un espacio de interacción sumamente amigable, creativo e innovador, en el cual no existen limitaciones en cuanto a espacio y tiempo para que los participantes una vez se registren, obtengan la información contentiva en cada unidad, con el apoyo de videos, lecturas y presentaciones con contenido resumido que hacen más fácil la comprensión del contenido, pretendiendo que puedan transferir esos conocimientos al resto de los cursos y a su desempeño profesional o como parte de su vida diaria.

\section{REFERENCIAS}

Autismo en voz alta. Bienvenidos a autismo en voz alta. [Página en línea]. Disponible: http://autismoenvozalta.com/index.php/ es/ [Consulta: 2016, febrero 25]

Dorrego, E. y García, A. (1993). Dos modelos para la producción y evaluación de materiales instruccionales. Caracas: Universidad Central de Venezuela, Facultad de Humanidades y Educación

East y Evans (2013). Guía práctica de necesidades educativas especiales. Madrid: Morata

Gavidia, V. (2003). La educación para la salud en los manuales escolares españoles. Revista Española de Salud Pública. [Revista en li-

nea].Disponible:http://scielo.isciii.es/scie lo.php?pid=s113557272003000200010\& script=sci_arttext [Consulta: 2016, marzo 12]

Ley Orgánica de Ciencia, Tecnología e Innovación. Gaceta Oficial de la República Bolivariana de Venezuela, 38.588 Diciembre 20, 2006

Lozano, A. y Burgos, J. (2010). Tecnología Educativa en un modelo de educación a distancia centrado en la persona. México: Limusa

Moore, M. y Kearsley, G. (1996). Distance education: A Systems View. Belmont, CA.: Wadsworth

Müller, G. (2003). Un modelo de desarrollo basado en el estudio de necesidades. $\mathrm{Vi}$ cerrectorado de Extensión. (3) 1.

Organización Mundial de la Salud. (1998). Promoción de la salud. Glosario. [Documento en línea]. Disponible:

http://www.msssi.gob.es/profesionales/ saludPubli-

ca/prevPromocion/docs/glosario.pdf [Consulta: 2016, mayo 12]

¿Qué es Moodle? [Documento en línea.] Disponible:

http://www.entornos.com.ar/moodle[Co nsulta: 2016, febrero 25]

Szczurek, M. (1989). La estrategia instruccional. Revista de Investigación y Postgrado, $4(2)$

Universidad Pedagógica Experimental Libertador. (2016). Manual de Trabajos de Grado de Especialización y Maestría y Tesis Doctorales. Caracas: Autor 